\title{
Fototrampeo de mamíferos en la Sierra Nanchititla, México: abundancia relativa y patrón de actividad
}

\author{
Octavio Monroy-Vilchis ${ }^{1 *}$, Martha M. Zarco-González ${ }^{1}$, Clarita Rodríguez-Soto ${ }^{1}$, \\ Leroy Soria-Díaz ${ }^{1} \&$ Vicente Urios ${ }^{2}$ \\ 1. Estación Biológica Sierra Nanchititla, Universidad Autónoma del Estado de México. Instituto Literario 100, Centro, \\ C.P. 50000, Toluca, México; omv@uaemex.mx, tavomonroyvilchis@gmail.com, marthamarielazg@yahoo.com.mx, \\ crs115@hotmail.com, leroysoria@hotmail.com \\ 2. Centro Iberoamericano de la Biodiversidad, Universidad de Alicante, Apartado 99.03080 Alicante, España; \\ vicenteurios@yahoo.es \\ * Correspondencia.
}

Recibido 22-III-2010. C Corregido 22-IX-2010. Aceptado 22-X-2010.

\begin{abstract}
Mammals' camera-trapping in Sierra Nanchititla, Mexico: relative abundance and activity patterns. Species conservation and their management depend on the availability of their population behavior and changes in time. This way, population studies include aspects such as species abundance and activity pattern, among others, with the advantage that nowadays new technologies can be applied, in addition to common methods. In this study, we used camera-traps to obtain the index of relative abundance and to establish activity pattern of medium and large mammals in Sierra Nanchititla, Mexico. The study was conducted from December 2003 to May 2006, with a total sampling effort of 4305 trap-days. We obtained 897 photographs of 19 different species. Nasua narica, Sylvilagus floridanus and Urocyon cinereoargenteus were the most abundant, in agreement with the relative abundance index (RAI, number of independent records/100 trap-days), and according to previous studies with indirect methods in the area. The activity patterns of the species showed that $67 \%$ of them are nocturnal, except Odocoileus virginianus, Nasua narica and others. Some species showed differences with previously reported patterns, which are related with seasonality, resources availability, organism sex, principally. The applied method contributed with reliable data about relative abundance and activity patterns. Rev. Biol. Trop. 59 (1): 373-383. Epub 2011 March 01.
\end{abstract}

Key words: abundance, camera-traps, mammals, nocturnal habits.

La abundancia relativa de los mamíferos es un indicador de la situación poblacional y su evaluación en diferentes tiempos o espacios evidencia su posible variación espacial y temporal. Este parámetro, al igual que el patrón de actividad pueden contribuir a la propuesta de estrategias para la conservación de las especies (Walker et al. 2000). La estimación de la abundancia para el caso de mamíferos tanto medianos como grandes es difícil y costosa debido a sus hábitos nocturnos y evasivos además por lo general, se encuentran en bajas densidades, por lo anterior es recomendable el cálculo de índices de abundancia relativa (Sutherland 1996). Los índices obtenidos son el resultado del muestreo de una fracción de la población y se expresan como el número de individuos contados por unidad de muestreo (Maffei et al. 2002, O'Brien et al. 2003, Yasuda 2004, Rovero \& Marshall 2009).

La técnica de fototrampeo en estudios poblacionales de mamíferos (no roedores ni quirópteros) es una herramienta confiable y no invasiva (Silveira et al. 2003, Pinto de Sá Alves \& Andriolo 2005, Monroy-Vilchis et al. 2009), que contribuye a su estudio y ofrece ciertas 
ventajas en comparación con otros métodos como el trampeo directo y la telemetría, ya que estos últimos son más costosos, proporcionan un reducido número de registros, además de que alteran el comportamiento de los individuos (Krausman 2002). La eficiencia del fototrampeo ha sido demostrada en trabajos como el de Silveira et al. (2003) en Brasil, quiénes compararon los índices de abundancia relativa obtenidos a través de diversos métodos, y concluyeron que el fototrampeo es el más apropiado, ya que permite una rápida evaluación del estatus de conservación de la vida silvestre. Además, a partir de las fotografías es posible registrar especies crípticas, que se mueven largas distancias o que están en bajas densidades (Srbek-Araujo \& García 2005, Monroy-Vilchis et al. 2009). Sus ventajas incluyen la precisión en la identificación a nivel específico y frecuentemente individual, una eficiencia de detección similar en animales diurnos y nocturnos y la confirmación de especies cuyas huellas no se diferencian (Maffei et al. 2002, Trolle \& Kéry 2003, Wallace et al. 2003, Karanth et al. 2004, Maffei et al. 2004, Di Bitetti et al. 2006, Soisalo \& Cavalcanti 2006, Dillon \& Kelly 2008, Maffei \& Noss 2008).

Las cámaras son útiles para evaluar patrones de actividad (Maffei et al. 2002, Rumiz et al. 2002, Pinto de Sá Alves \& Andriolo 2005, Monroy-Vilchis et al. 2009) y uso de hábitat (Bowkett et al. 2007), así como para realizar estimaciones de densidad (Trolle \& Kéry 2003, Karanth et al. 2004, Maffei et al. 2004, Di Bitetti et al. 2006, Soisalo \& Cavalcanti 2006, Dillon \& Kelly 2008, Maffei \& Noss 2008, Trolle 2008). La frecuencia de captura ha sido utilizada como un índice de abundancia relativa (IAR) evidenciando su correlación con las densidades absolutas, además de su relativa facilidad de aplicación (Maffei et al. 2002, O'Brien et al. 2003, Silveira et al. 2003, De Almeida et al. 2004, Yasuda 2004, Kelly 2008, Monroy-Vilchis et al. 2009, Rovero \& Marshall 2009). De esta manera los objetivos del presente estudio fueron evaluar la abundancia relativa y el patrón de actividad de los mamíferos medianos y grandes de la Reserva Natural Sierra Nanchititla.

\section{MATERIALES Y MÉTODOS}

Área de estudio: La Reserva Natural Sierra Nanchititla (RNSN) tiene una extensión de 66338ha. Se localiza en la región fisiográfica de la Cuenca del Balsas, en el suroeste del Estado de México. Sus coordenadas geográficas son $18^{\circ} 45^{\prime} 13^{\prime \prime}-19^{\circ} 04^{\prime} 22^{\prime \prime} \mathrm{N}$ y $100^{\circ} 16^{\prime} 03^{\prime \prime}$ $100^{\circ} 36^{\prime} 49^{\prime \prime}$ W. Es una de las zonas más diversas y ecológicamente importantes dentro del Estado, donde se han registrado 53 especies de mamíferos. La mayor parte del área (40\%) está entre 410 y $1000 \mathrm{msnm}$, el $32 \%$ presenta altitudes entre 1000 y $1400 \mathrm{~m}$ y el restante $28 \%$ va de los 1400 a los $2080 \mathrm{~m}$. De los tipos de vegetación presentes en la RNSN, la selva baja caducifolia ocupa la mayor extensión $\left(209.98 \mathrm{~km}^{2}, 31.5 \%\right)$, seguida por el bosque de latifoliadas, que incluye el bosque de encino, mesófilo de montaña y de galería $\left(91.13 \mathrm{~km}^{2}\right.$, $13.7 \%)$ y el bosque de pino-encino $\left(68.53 \mathrm{~km}^{2}\right.$, $10.3 \%$ ). Sin embargo, un área considerable de la Sierra ha sido transformada en mocultivos de maíz $\left(259.66 \mathrm{~km}^{2}, 39.1 \%\right)$ y zonas de pastizal $\left(35.3 \mathrm{~km}^{2}, 5.3 \%\right)$ (Rubio-Rodríguez 2009).

El muestreo se llevó a cabo durante el periodo de diciembre de 2003 a mayo de 2006. Sobre caminos, veredas y barrancos que abarcaron todos los tipos de vegetación de la zona, se colocaron 17 estaciones de trampeo con una trampa-cámara cada una, con el fin de aumentar la probabilidad de registro de las diferentes especies. Las trampas-cámara utilizadas son sistemas de detección fotográfica automática, marca CamTrakker ${ }^{\circledR}$ que operan a partir de un censor infrarrojo pasivo, el circuito fue programado para permanecer activo las 24 horas y con un retraso mínimo de 0.3 minutos entre cada disparo, se revisaron una vez al mes y su posición fue georeferenciada con un geoposicionador (Garmin colorado 400). Se usaron películas fotográficas de $35 \mathrm{~mm}$ con 400 ASA, en cada fotografía se imprimieron la hora y la fecha. Los datos fueron procesados en el programa ArcView 3.2 (Environmental System 
Research Institute 2000). Las cámaras estuvieron activas un mes cada cuatro meses durante todo el periodo de muestreo, con el fin de considerar tanto la temporada de lluvias como de secas. El esfuerzo total de muestreo fue la suma de los días-trampa que cada trampa-cámara permaneció activa.

Las especies fotografiadas fueron identificadas por comparación con base en literatura especializada (Hall 1981, Emmons \& Feer 1990, Reid 1997, Ceballos \& Oliva 2005). La clasificación y nomenclatura utilizadas se basaron en Ramírez et al. (2005). Se calculó el número de registros fotográficos independientes adquiridos por cada 100 días-trampa como un índice de abundancia relativa (O'Brien et al. 2003). El esfuerzo de muestreo de 100 díastrampa es una unidad de estandarización para comparar los datos con otros estudios (Monroy-Vilchis et al. 2009). Con el fin de estimar con mayor precisión la abundancia al evitar contar varias veces al mismo individuo, sólo se consideraron como registros fotográficos independientes los siguientes casos: 1) fotografías consecutivas de diferentes individuos, 2) fotografías consecutivas de individuos de la misma especie separadas por más de $24 \mathrm{~h}$, este criterio fue aplicado cuando no era claro si una serie de fotografías correspondían al mismo individuo, de modo que las fotografías tomadas dentro del mismo periodo de $24 \mathrm{~h}$ se consideraron como un sólo registro, 3 ) fotografías no consecutivas de individuos de la misma especie. En el caso de las especies gregarias, en las fotografías en las que se observó más de un individuo, el número de registros independientes considerado fue igual al número de individuos observados en la misma.

Para determinar el patrón de actividad de las especies se obtuvieron al menos 11 registros fotográficos independientes con la hora visible, que se ha considerado como el número mínimo para describir el patrón de actividad (Maffei et al. 2002, Monroy-Vilchis et al. 2009). Posteriormente se cuantificó el porcentaje de registros obtenidos en intervalos de dos horas. Las fotografías se consideraron de día cuando se observaba luz solar y de noche cuando no la había. Para hacer más preciso el análisis de las especies crepusculares, el amanecer se consideró entre 06:00-08:00h y el atardecer entre 18:00-20:00h.

\section{RESULTADOS}

El esfuerzo de muestreo fue de 4305 días-trampa y se obtuvieron 897 fotografías de 19 especies de mamíferos. Éstas especies se ubican en diez familias y seis órdenes, el orden mejor representado fue Carnivora con cuatro familias y 12 especies (Cuadro 1). Del total de fotografías, 640 (71\%) fueron clasificadas como registros independientes, de las cuales el $67 \%$ se obtuvieron de noche.

De acuerdo con el IAR las especies más abundantes fueron Nasua narica, Sylvilagus floridanus y Urocyon cinereoargenteus. Las especies Odocoileus virginianus, $U$. cinereoargenteus y $N$. narica, fueron registradas en más estaciones de trampeo (Cuadro 1).

Del total de fotografías independientes, el $60 \%$ presentaban la hora visible y fueron usadas para analizar los patrones de actividad. Los registros de $N$. narica se presentaron durante todo el día, pero particularmente entre 18:0020:00h. Didelphis virginiana se observó como una especie nocturna, al igual que las dos especies del género Sylvilagus y U. cinereoargenteus que mostraron una tendencia hacia hábitos nocturnos, con muy pocos registros durante el día. O. virginianus no mostró tendencias hacia algún horario en particular, fue activo tanto de día como de noche, al igual que Pecari tajacu. Sciurus aureogaster se observó como una especie completamente diurna con actividad entre las 06:00-18:00h (Fig. 1).

\section{DISCUSIÓN}

Los métodos tradicionales para los estudios poblacionales de mamíferos grandes como el recorrido de trayectos, conteos en vehículo y las técnicas de marcaje-recaptura requieren de mucho tiempo de trabajo y están limitados a hábitat con alta visibilidad (Roberts et al. 


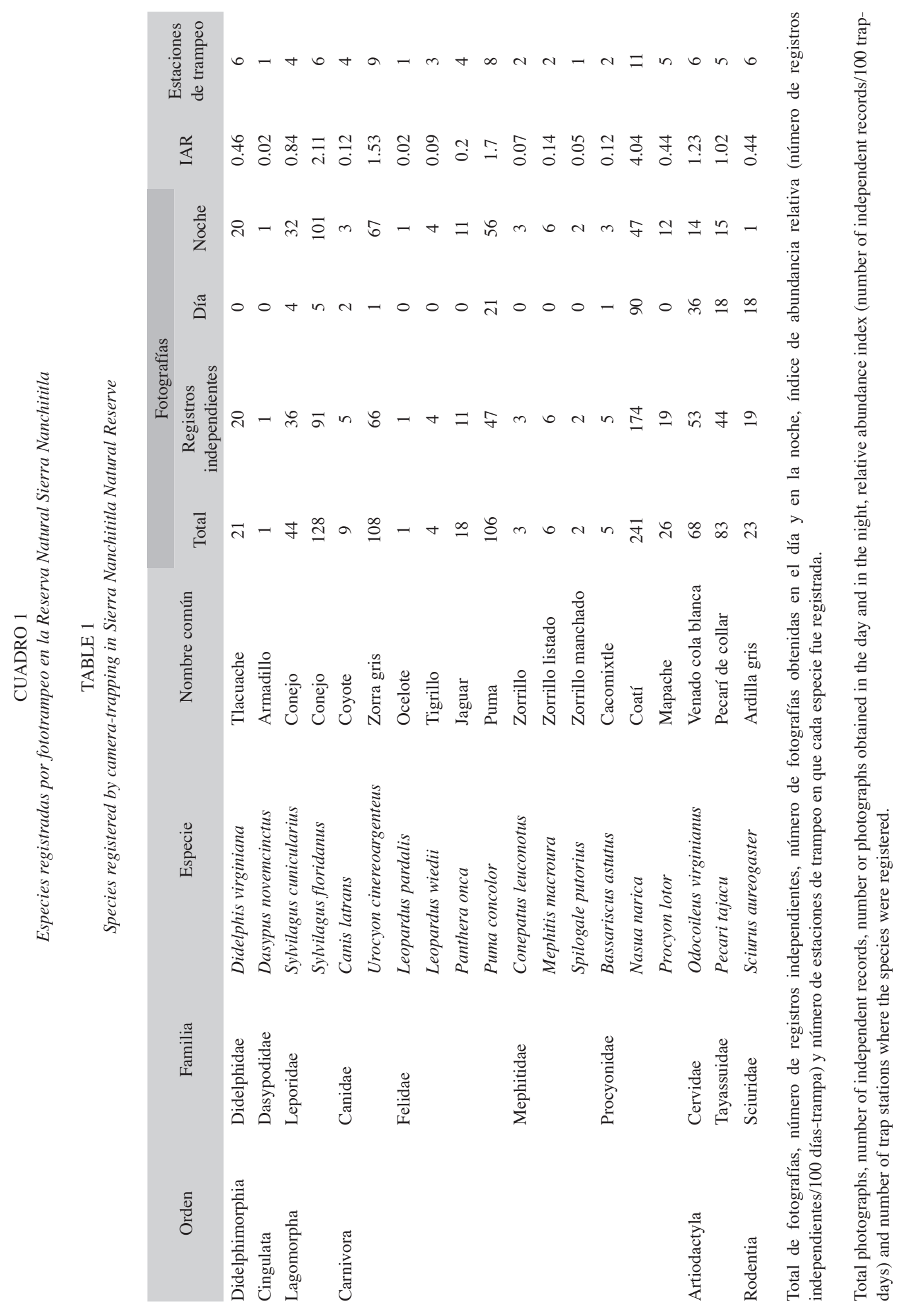



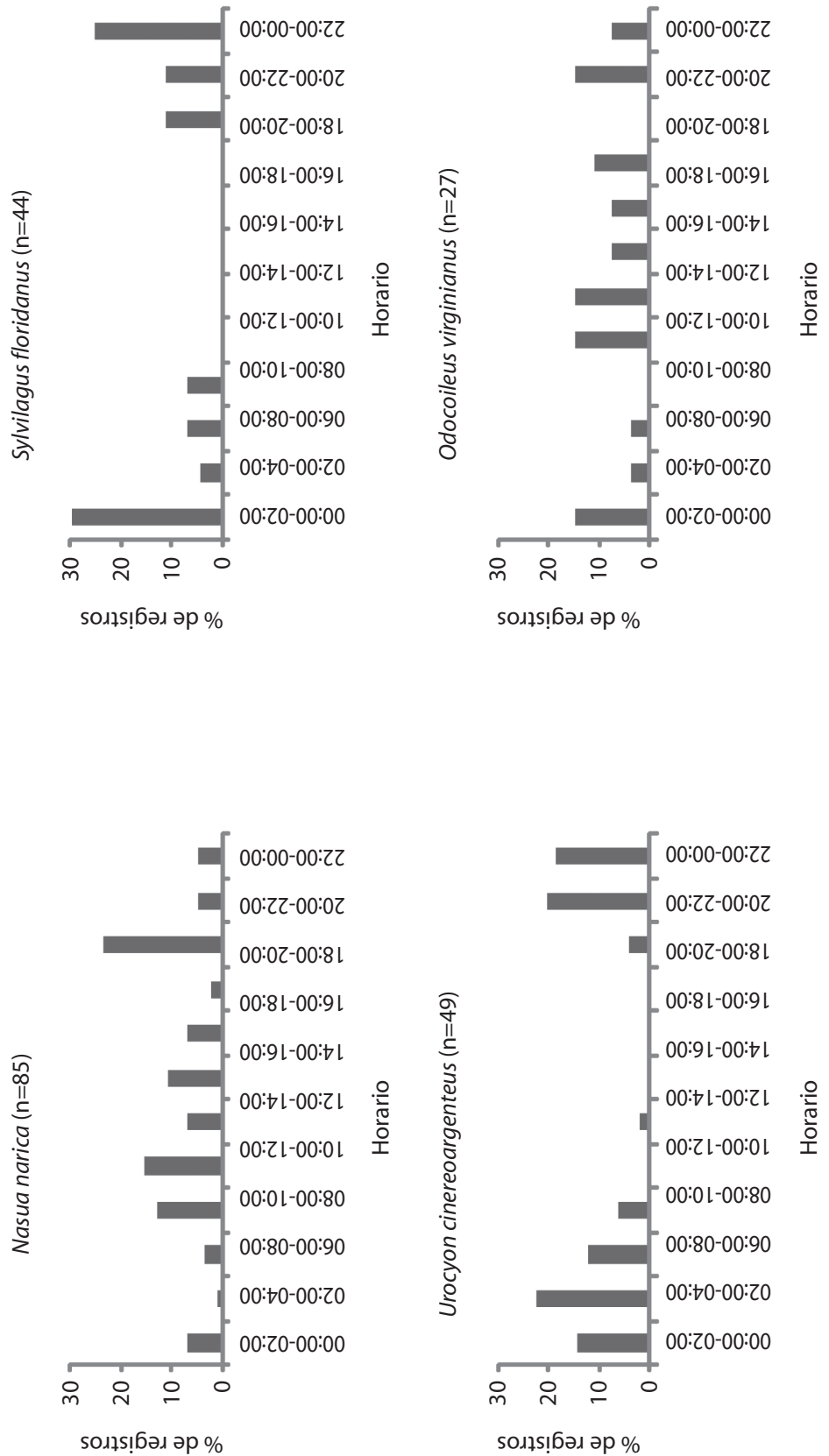

Fig. 1. Patrones de actividad de las especies más abundantes en la RNSN.

Fig. 1. Activity patterns of most abundant species in SNNR. 
2006), distintos a las condiciones del área de estudio, ya que se trata de una zona muy escarpada; por lo que el uso de trampas-cámara es una alternativa más precisa que los métodos anteriores.

A partir del fototrampeo se obtuvieron datos de las especies de mamíferos medianos y grandes que se han registrado hasta ahora en la RNSN, excepto Puma yagouaroundi. Con ésta técnica fue posible obtener además del registro de los animales, información sobre diversos aspectos ecológicos como abundancia relativa, patrón de actividad e incluso información que puede ser útil para hacer inferencias sobre cuestiones reproductivas, por ejemplo registros de hembras con crías, así como parejas de hembras y machos adultos (Monroy-Vilchis et al. 2009).

Actualmente, existe un debate sobre la validez de la frecuencia de captura como un índice de abundancia relativa, algunos autores han argumentado que el número de fotografías obtenidas de una especie depende de la probabilidad de detección, más que de la abundancia de la misma (Tobler et al. 2008, Harmsen et al. 2010). Sin embargo, Kelly (2008) establece que la consistencia que generalmente se observa entre los índices obtenidos en distintos años, como es el caso de este estudio, puede aumentar la confiabilidad de la frecuencia de captura para estimar la abundancia relativa de las especies. De la misma manera, Rowcliffe \& Carbone (2008) mencionan que, controlando algunas variables que podrían influir en los índices de captura, es posible extraer la abundancia basada en frecuencias de fototrampeo. En esta investigación se colocaron las trampascámara en todos los tipos de vegetación de la zona, en sitios con vegetación tanto abierta como cerrada, en caminos ya establecidos que son transitados por humanos y en veredas que son frecuentemente utilizadas por mamíferos medianos y pequeños, además de hacerlo en ambas temporadas (lluvias y secas); todo esto con el fin de aumentar la probabilidad de detección de las diferentes especies.

La influencia de las cámaras sobre el comportamiento de las especies tampoco es un factor que pueda considerarse una desventaja de la técnica, ya que se han encontrado resultados contradictorios, por ejemplo Gompper et al. (2006) sugieren que los coyotes evitan las trampas-cámara, sin embargo, Kelly \& Holub (2008) mencionan que en el estudio que realizaron en Virginia esta especie fue una de las que presentaron un éxito de trampeo más alto, junto con el venado cola blanca, el mapache y la zorra gris. De la misma manera, en Sonora, Pina et al. (2004) encontraron que el venado cola blanca, la zorra gris y el coyote fueron las especies registradas con mayor frecuencia por las trampas-cámara. En el caso particular de Dasypus novemcinctus varios autores coinciden en que el fototrampeo no es la técnica más adecuada para analizar su abundancia (Weckel et al. 2006, Harmsen et al. 2010), considerando esto, así como el bajo número de registros obtenidos en este estudio, es recomendable aplicar otras técnicas para esta especie.

De acuerdo con los IAR calculados, las especies más abundantes son $N$. narica, $S$. floridanus y $U$. cinereoargenteus, lo cual es congruente con lo observado en campo con respecto a los rastros y avistamientos en estudios previos en la zona (Monroy-Vilchis et al. 2008). La abundancia de S. floridanus puede estar influida por el cambio de uso de suelo de vegetación primaria a campos de cultivo y pastizales, ya que, como se ha visto en otros estudios, el crecimiento poblacional de esta especie se ve favorecido con la introducción de estos tipos de cobertura (Bock et al. 2006). Por otro lado, $S$. cunicularius, aunque no fue de las especies más abundantes en este caso, si se encuentra entre las primeras siete, lo cual es relevante si se considera que se trata de una especie endémica de México, y que en otras zonas de su distribución como La Malinche, se ha encontrado con una abundancia baja (González et al. 2007), por lo que información sobre su estatus es particularmente importante para su conservación.

La mayor cantidad de fotografías se obtuvo entre las 18:00 y 02:00h, mientras que se registraron más especies entre las 20:00 y 08:00h. Esto es debido a que, con excepción de los primates, la mayoría de los mamíferos 
neotropicales son predominantemente nocturnos (Srbek-Araujo \& García 2005).

Con respecto al análisis del patrón de actividad, Van Schaik \& Griffiths (1996) mencionan que el tamaño corporal se relaciona con el mismo, de manera que los animales más grandes, dados sus requerimientos energéticos mayores, deben forrajear durante más tiempo, por lo que son activos tanto de día como de noche; mientras que los hábitos nocturnos de los animales pequeños $(<10 \mathrm{~kg})$ se relacionan con la evasión del riesgo de depredación. Sin embargo, algunos de los resultados de este estudio contradicen esta conclusión, ya que especies de menos de $10 \mathrm{~kg}$, como el coatí y la ardilla fueron activos principalmente durante el día, por lo que es más probable que otros factores tanto externos (temperatura, humedad, disponibilidad de recursos, etc.), como inherentes a los individuos de cada especie (sexo, edad, estatus reproductivo, etc.), tengan mayor influencia sobre su patrón de actividad.

D. virginiana por lo general se ha considerado una especie completamente nocturna (Emmons \& Feer 1990). Ceballos \& Galindo (1984) establecen que la mayor actividad se presenta entre 23:00-02:00h , aunque no menciona cual fue el método usado para determinar tal dato. Mediante un estudio con radiotelemetría, González et al. (1992) describen para la especie un aumento en la actividad durante el atardecer. En este estudio el pico de actividad se presentó entre las 22:00-00:00h. Las diferencias entre los trabajos anteriores puede deberse a los cambios en la temperatura ambiental de las distintas zonas de estudio, la cual se ha observado como un factor determinante para la actividad del tlacuache, negativamente relacionada con el tiempo de forrajeo (Kanda et al. 2005).

La actividad de $U$. cinereoargenteus se ha descrito como principalmente nocturna (Reid 1997), como ocurre en este estudio. Sin embargo, González et al. (1992) encontraron que existe una influencia estacional sobre el patrón de actividad, de manera que se presenta un aumento de la misma durante el día en la temporada otoño-invierno, lo cual atribuyen a la diferencia en la disponibilidad de recursos alimentarios, ya que durante dicha temporada disminuye la fructificación de las especies vegetales que consume, por lo que es necesario un tiempo y esfuerzo mayor para la captura de otras presas.

Valenzuela \& Ceballos (2000) estudiaron la actividad de $N$. nasua en relación con la estacionalidad climática, mediante el uso de radiotelemetría; encontraron que los machos son más activos durante la noche que los grupos (hembras y juveniles), en este estudio se observó un comportamiento similar. Durante el día, tanto los machos como los grupos son activos, pero existen algunas diferencias, los machos no fueron activos de 06:00-10:00h ni entre 16:00-18:00h y presentaron un aumento de registros de 12:00-14:00h, horario en el que la actividad de los grupos se mantiene baja, esto último coincide con los resultados de un estudio realizado por Costa et al. (2009) a partir de la observación directa de grupos de $N$. nasua, donde se observó que los periodos de inactividad se concentran al medio día durante la estación seca, mientras que durante las lluvias la actividad está uniformemente distribuida a lo largo del día. Estos autores sugieren que este cambio estacional puede estar relacionado con una estrategia de ahorro de energía, ya que durante la temporada seca los recursos alimenticios son escasos. En la mayoría de las especies, las diferencias en los patrones entre las hembras y los machos se deben a que los requerimientos energéticos y las limitaciones para la obtención de recursos son distintos. El requerimiento principal de las hembras es el alimento, mientras que los machos tienen que desplazarse para encontrar, además de alimento, parejas reproductivas. Las hembras tienen mayores requerimientos energéticos que los machos, lo que implica mayor actividad durante más horas (Valenzuela \& Ceballos 2000). El pico de actividad durante el atardecer se observó tanto en machos como en grupos de hembras con crías. El patrón general de la especie implica hábitos predominantemente diurnos, lo que coincide con Emmons \& Feer (1990), Reid (1997) y Pinto de Sá Alves \& Andriolo (2005). 
Para $O$. virginianus se han mencionado picos de actividad en la mañana de 05:00-08:00h, en la tarde de 17:00-19:00h y por la noche de 22:00-01:00h en ambientes templados de la Sierra Madre Occidental (Galindo \& Weber 2005). Sin embargo, en este estudio se observa un desplazamiento al existir registros de las 00:00-02:00h, además de que hay periodos que no coinciden con los citados anteriormente: de 08:00-12:00h y entre 20:00-22:00h. Por otro lado, es clara la falta de actividad crepuscular, contrario a lo mencionado por Galindo-Leal \& Weber (1998) y Gallina et al. (2005) que mencionan un aumento en la actividad durante el crepúsculo, específicamente de 05:00-09:00h y de 17:00-21:00h.

Tobler et al. (2009) mencionan que $P$. tajacu es una especie completamente diurna, sin embargo, en este estudio esta especie también fue registrada durante la noche, lo que coincide con Emmons \& Feer (1990) y Reid (1997). Estas diferencias pueden deberse a que el estudio de Tobler et al. (2009) se llevó a cabo en un bosque tropical lluvioso de Perú, en el que las condiciones ambientales son más estables a lo largo del año, en comparación con la marcada estacionalidad que se presenta en la RNSN, ya que se ha visto que debido al aumento de la temperatura ambiental durante el día, los pecaríes en zonas como esta tienden a ser activos también durante la noche (Emmons \& Feer 1990).

El comportamiento completamente diurno de $S$. aureogaster corresponde con lo mencionado anteriormente por otros autores (Emmons \& Feer 1990). Esto es muy interesante y es importante diseñar un estudio para entender mejor esta dinámica.

En algunas especies, los datos obtenidos no fueron suficientes para determinar los patrones de actividad, sin embargo, se observaron algunas tendencias. En el caso del Bassaricus astutus se obtuvieron fotografías tanto de día como de noche, aunque es una especie comúnmente considerada como nocturna (Reid 1997). Canis latrans también fue registrado durante el día y la noche. Conepatus leuconotus, Mephitis macroura, D. novemcinctus, Leopardus wiedii y L. pardalis sólo se fotografiaron durante la noche. Según lo mencionado, Procyon lotor es de tendencia crepuscular, con un pico de forrajeo a la media noche (Valenzuela 2005), en este trabajo se comprobó lo anterior, ya que en varias ocasiones se registraron grupos de mapaches entre las 00:00-03:00h.

De esta manera fue posible generar información acerca de la abundancia relativa de los mamíferos medianos y grandes de la RNSN, así como sus patrones de actividad en los que se observa una tendencia general hacia hábitos nocturnos; esta información puede contribuir a la planeación del manejo y conservación de dichas especies.

\section{AGRADECIMIENTOS}

Agradecemos al pueblo mexicano por financiar este estudio a través de la Universidad Autónoma del Estado de México con los proyectos FE012/2006, FE09/2007, FE014/20072009; El CONACYT con el proyecto 101254 y el PROMEP con el proyecto 103.5/10/0942. La Comisión Estatal de Parque Naturales y de la Fauna nos permitió el acceso al Parque. Estudiantes de la Estación Biológica Sierra Nanchititla nos ayudaron en el trabajo de campo.

\section{RESUMEN}

La conservación de las especies y su manejo adecuado dependen de la disponibilidad de información sobre sus poblaciones, por ello es importante estudiar aspectos como la abundancia y el patrón de actividad. En esta investigación se utilizaron trampas-cámara para obtener índices de abundancia relativa y establecer el patrón de actividad de los mamíferos medianos y grandes de la Sierra Nanchititla, México. El trabajo se llevó a cabo durante el periodo de diciembre de 2003 a mayo de 2006, con un esfuerzo total de 4305 días-trampa. Se obtuvieron 897 fotografías de 19 especies, las más abundantes fueron: Nasua narica, Sylvilagus floridanus y Urocyon cinereoargenteus, de acuerdo con el índice de abundancia relativa (IAR, número de registros independientes/100 días trampa), coincidiendo con estudios basados en métodos indirectos. El patrón de actividad de las especies registradas mostraron que el $67 \%$ son de hábitos nocturnos. Algunas especies mostraron diferencias con los patrones mencionados por otros autores, las cuales se relacionan principalmente con la estacionalidad, la disponibilidad de recursos y el sexo de los individuos. 
Palabras clave: abundancia, hábitos nocturnos, mamíferos, trampas-cámara.

\section{REFERENCIAS}

Bock, C.E., Z.F. Jones \& J.H. Bock. 2006. Abundance of cottontails (Sylvilagus) in an exurbanizing Southwestern savanna. Southwest. Nat. 51: 352-357.

Bowkett, A., F. Rovero \& A. Marshall. 2007 The use of camera-trap data to model habitat use by antelope species in the Udzungwa Mountain forests, Tanzania. Afr. J. Ecol. 46: 479-487.

Ceballos, G. \& C. Galindo. 1984. Mamíferos silvestres de la Cuenca de México. Limusa, D. F., México.

Ceballos, G. \& G. Oliva. 2005. Los mamíferos silvestres de México. Fondo de Cultura Económica, CONABIO, Hong Kong, China.

Costa, E., R. Mauro \& S. Silva. 2009. Group composition and activity patterns of brown-nosed coatis in savanna fragments, Mato Grosso do Sul, Brazil. Braz. J. Biol. 69: 985-991.

De Almeida, A.T., L. Silveira \& J.A. Felizola. 2004. Niche separation between the maned wolf (Chrysocyon brachyurus), the crab-eating fox (Dusicyon thous) and the hoary fox (Dusicyon vetulus) in central Brazil. J. Zool. 262: 99-106.

Di Bitetti, M., A. Paviolo \& C. De Angelo. 2006. Density, habitat use and activity patterns of ocelots (Leopardus pardalis) in the Atlantic Forest of Misiones, Argentina. J. Zool. 270: 153-163.

Dillon, A. \& M.J. Kelly. 2008. Ocelot home range, overlap and density: comparing radiotelemetry with camera trapping. J. Zool. 275: 391-398.

Emmons, L. \& F. Feer. 1990. Neotropical Rainforest Mammals. A Field Guide. The University of Chicago, Chicago, EEUU.

Environmental Systems Research Institute. 2000. ArcView GIS (Version 3.2a). Environmental Systems Research Institute, Redlands, California, EEUU.

Galindo, C. \& M. Weber. 2005. Venado cola blanca, p. $517-$ 521. In G. Ceballos \& G. Oliva (eds.). Los mamíferos silvestres de México. Fondo de Cultura Económica y CONABIO, Hong Kong, China.

Galindo-Leal, C. \& M. Weber. 1998. El venado de la Sierra Madre Occidental. Ecología, manejo y conservación. EDICUSA-CONABIO, D.F., México.
Gallina, S., P. Corona-Zárate \& J. Bello. 2005. El comportamiento del venado cola blanca en zonas semiáridas del Noreste de México, p. 193-203. In V. SánchezCordero \& R.A. Medellín (eds.). Contribuciones mastozoológicas en homenaje a Bernardo Villa. Instituto de Biología de la UNAM-Instituto de Ecología de la UNAM-CONABIO, D. F., México.

Gompper, M.E., R.W. Kays, J.C. Ray, S.D. Lapoint, D.A. Bogan \& J.R. Cryan. 2006. Comparison of noninvasive techniques to survey carnivore communities in Northeastern North America. Wildlife. Soc. B. 34: 1142-1151.

González, G., V. Sánchez, L. Íñiguez, E. Santana \& T. Fuller. 1992. Patrones de actividad de coyote (Canis latrans), la zorra gris (Urocyon cinereoargenteus) y el tlacuache (Didelphis virginiana) en la Sierra de Manantlán, Jalisco. An. Inst. Biol. Univ. Nac. Auton. Mex. Ser. Zool. 63: 293-299.

González, J., C. Lara, J. Vázquez \& M. Martínez-Gómez. 2007. Demography, density, and survival of an endemic and near threatened cottontail Sylvilagus cunicularius in central Mexico. Acta. Theriol. 52: 299-305.

Hall, E.R. 1981. The Mammals of North America. Wiley, Nueva York, EEUU.

Harmsen, B., R. Foster, S. Silver, L. Ostro \& P. Doncaster. 2010. Differential use of trails by forest mammals and the implications for camera-trap studies: a case study from Belize. Biotropica 42: 126-133.

Kanda, L.L., T.K. Fuller \& K.D. Friedland. 2005. Temperature sensor evaluation of opossum winter activity. Wildlife. Soc. B. 33: 1425-1431.

Karanth, K.U., R.S. Chundawat, J.D. Nichols \& N.S. Kumar. 2004. Estimation of tiger densities in the tropical dry forests of Panna, Central India, using photographic capture-recapture sampling. Anim. Conserv. 7: 285-290.

Kelly, M. 2008. Design, evaluate, refine: camera trap studies for elusive species. Anim. Conserv. 11: 182-184.

Kelly, M.J. \& E.L. Holub. 2008. Camera trapping of carnivores: Trap success among camera types and across species, and habitat selection by species, on Salt Pond Mountain, Giles County, Virginia. Northeast. Nat. 15: 249-262.

Krausman, P. 2002. Introduction to wildlife management. Prentice Hall, Nueva Jersey, EEUU.

Maffei, L., E. Cuellar \& A. Noss. 2004. One thousand jaguars (Panthera onca) in Bolivia's Chaco? camera 
trapping in the Kaa-Iya National Park. J. Zool. 262: 295-304.

Maffei, L., E. Cuellar \& J. Noss. 2002. Uso de trampascámara para la evaluación de mamíferos en el ecotono Chaco-Chiquitanía. Rev. Bol. Ecol. 11: 55-65.

Maffei, L. \& A.J. Noss. 2008. How small is too small? camera trap survey areas and density estimates for ocelots in the Bolivian Chaco. Biotropica 40: 71-75.

Monroy-Vilchis, O., C. Rodríguez-Soto. M. Zarco-González \& V. Urios. 2009. Cougar and jaguar habitat use and activity patterns in Central Mexico. Anim. Biol. 59: $145-157$.

Monroy-Vilchis, O., L. Cabrera, P. Suárez, M. ZarcoGonzález, C. Rodríguez-Soto \& V. Urios. 2008. Uso tradicional de vertebrados silvestres en la Sierra Nanchititla, México. Interciencia 33: 308-313.

O’Brien, T., M. Kinnaird \& H. Wibisono. 2003. Crouching tigers, hidden prey: Sumatran tiger and prey populations in a tropical landscape. Anim. Conserv. 6: 131-139.

Pina, G.P.L., R.A.C. Gamez \& C.A.L. González. 2004. Distribution, habitat association and activity patterns of medium and large sized mammals of Sonora, Mexico. Nat. Area. J. 24: 354-357.

Pinto de Sá Alves, L.C. \& A. Andriolo. 2005. Camera traps used on the mastofaunal survey of Araras Biological Reserve, IEF-RJ. Rev. Bras. Zootecn. 2: 231-246.

Ramírez, J., J. Arroyo \& A. Castro. 2005. Estado actual y relación nomenclatural de los mamíferos terrestres de México. Acta. Zool. Mex. 21: 21-82.

Reid, F.A. 1997. A field guide to the mammals of Central America and Southeast Mexico. Oxford University, Nueva York, EEUU.

Roberts, C.W., B.L. Pierce, A.W. Braden, R.R. Lopez, N.J. Silvy, P.A. Frank \& D. Ranzom. 2006. Comparison of camera and road survey estimates for white-tailed deer. J. Wildlife Manage. 70: 263-267.

Rovero, F. \& A. Marshall. 2009. Camera trapping photographic rate as an index of density in forest ungulates. J. Appl. Ecol. 46: 1011-1017.

Rowcliffe, J. \& C. Carbone. 2008. Surveys using camera traps: are we looking to a brighter future? Anim. Conserv. 11: 185-186.
Rubio-Rodríguez, R. 2009. Regionalización biótica, abiótica y social del Parque Natural Sierra Nanchititla, México: propuesta de conservación. Tesis de Maestría, Facultad de Ciencias Agrícolas, Universidad Autónoma del Estado de México, Toluca, México.

Rumiz, D., A. Fuentes, K. Rivero, J. Santibáñez, E. Cuellar, R. Miserendino, I. Fernández, L. Maffei \& A. Taber. 2002. La biodiversidad de la Estancia San Miguelito, Santa Cruz-Bolivia: Una justificación para establecer reservas privadas de conservación. Instituto de Ecología, La Paz, Bolivia.

Silveira, L., A. Ja'como \& J. Diniz-Filhoa. 2003. Camera trap, line transect census and track surveys: a comparative evaluation. Biol. Conserv. 114: 351-355.

Soisalo, M.K. \& S.M.C. Cavalcanti. 2006. Estimating the density of a jaguar population in the Brazilian Pantanal using camera-traps and capture-recapture sampling in combination with GPS radio-telemetry. Biol. Conserv. 129: 487-496.

Srbek-Araujo, A.C. \& A. García. 2005. Is camera trapping an efficient method for surveying mammals in Neotropical forests? A case study in south-eastern Brazil. J. Trop. Ecol. 21: 121-125.

Sutherland, W. 1996. Ecological Census Techniques. Cambridge University, Cambridge, Gran Bretaña.

Tobler, M., S. Carrillo-Percastegui, R. Leite, R. Mares \& G. Powell. 2008. An evaluation of camera traps for inventorying large- and medium-sized terrestrial rainforest mammals. Anim. Conserv. 11: 169-178.

Tobler, M.W., S.E. Carrillo-Percastegui \& G. Powell. 2009. Habitat use, activity patterns and use of mineral licks by five species of ungulate in South-Eastern Peru. J. Trop. Ecol. 25: 261-270.

Trolle, M. 2008. Brazilian tapir density in the Pantanal: a comparison of systematic camera-trapping and linetransect surveys. Biotropica 40: 211-217.

Trolle, M. \& M. Kéry. 2003. Estimation of ocelot density in the Pantanal using capture-recapture analysis of camera- trapping data. J. Mammal. 84: 607-614.

Valenzuela, D. 2005. Mapache, p. 415-417. In G. Ceballos \& G. Oliva (eds.). Los mamíferos silvestres de México. Fondo de Cultura Económica y CONABIO, Hong Kong, China. 
Valenzuela, D. \& G. Ceballos. 2000. Habitat selection, home range and activity of the white nosed coati (Nasua nasua) in a Mexican Tropical Dry Forest. J. Mammal. 81: 810-819.

Van Schaik, C.P. \& M. Griffiths. 1996. Activity Periods of Indonesian Rain Forest Mammals. Biotropica 28: 105-112.

Walker, S., A. Novaro \& J. Nichols. 2000. Consideraciones para la estimación de abundancia de poblaciones de mamíferos. Mastozool. Neotrop. 7: 73-80.
Wallace, R.B., H. Gomez, G. Ayala \& F. Espinoza. 2003. Camera trapping for jaguar (Panthera onca) in the Tuichi Valley, Bolivia. Mastozool. Neotrop. 10: 133-139.

Weckel, M., W. Giuliano \& S. Silver. 2006. Jaguar (Panthera onca) feeding ecology: distribution of predator and prey through time and space. J. Zool. 270: 25-30.

Yasuda, M. 2004. Monitoring diversity and abundance of mammals with camera traps: a case study on Mount Tsukuba, central Japan. Mammal Study. 29: 37-46. 